\title{
A Reprise of the Properties of the Exotic Eclipsing Binary RT Lacertae
}

\author{
E. F. Milone \\ RAO, The University of Calgary, Physics $\&$ Astronomy Department, \\ 2500 University Drive, NW, Calgary, AB, T2N 1N4, Canada
}

\begin{abstract}
The 5.07-d eclipsing system RT Lacertae is at the interface of Algol systems and RS CVn-type systems. Three strong lines of evidence support its Algolid nature: gas streaming; the close proximity of the larger star to its Roche lobe; and evidence of circumstellar material. Supporting the stellar activity model are moderately successful, if nonunique, modeling of light curve anomalies and 'migration waves' with star spot models, and a general expectation based on similarities to RS CVnlike systems. Recent work by Lanza et al. (2002) provides support for the latter class of models, but other light curve analyses and non-optical passband studies are examined below and directions for the resolutions of the problems and paradoxes presented by the RT Lac system are provided. An observational campaign to acquire simultaneous spectroscopic, photometric, and polarimetric data is suggested.
\end{abstract}

\section{Introduction: Milestones in the Study of RT Lac}

RT Lacertae shows $\beta$ Lyrae type light curve variation (Luizet 1910, 1915). Mary Fowler (1920) produced the first light curve solution, based on Luizet's (1915) visual light curve. Spectral types, RV data, and a spectroscopic solution were first given by Joy (1931). Wachmann's (1935) photographic light curve showed asymmetry at maximum light and nearly equal depths, whereas the visual light curves of Luizet \& Nijland $(1917,1920)$ showed $\delta m_{v}$ between minima of $\sim 0.5$. Himpel's (1936) light curve demonstrated the variability of the minima: he found $\delta m_{v}=0.1$. A solution of the Wachmann data by Krat \& Nekrasova (1936) featured 'bright' and 'dark' sides of one of the components to account for brightness asymmetries. Beginning in 1965, the first published UBV data were obtained by Milone (1968) and Hall (1968) and analyzed by Milone (1977). These observations indicated a paradox: the deeper minimum (which all observers up to then had placed at zero phase) was bluer in $B-V$ and $U-B$ color indices. They also demonstrated unequal maxima (the ' $O$ 'Connell Effect') with the second maximum the brighter in all passbands, such that $\delta V_{\max }=-0.12 \pm 0.011$. Infrared $(J H K L)$ photometry was obtained and analyzed by Milone (1976). The assumption of a 'migrating wave' that traveled through the light curve over time permitted the removal of asymmetry to aid the analysis. In 1978, a team of observers in Turkey (e.g. Ibanoglu et al. 2001) began long-term optical monitoring of the system. Eaton \& Hall (1979) used the Wilson-Devinney program to obtain the first solution with Lagrangian surfaces for the stars. This work was 
not unproblematic: even the use of arbitrary distributions of spots on both stars failed to reproduce the distortions in the maxima fully, they did not reconcile a difference in secondary minimum depth in the two data sets $(1965,1968 \mathrm{~V}$ light curves), and, although they discuss the $U-V$ color index, they produced no modeled $B$ or $U$ light curves. Their work was nevertheless an important milestone. Huenemoerder (1985, 1988), and Huenemoerder \& Barden (1986) carried out spectrophotometry ( $\mathrm{H} \alpha, \mathrm{H} \beta, \mathrm{UV})$ that, while indicating the possibility of contributions from active regions, confirmed the presence of streaming effects. Popper (1991) considered that the star heretofore regarded as cooler (the more massive, designated 'star 1' here) to be the hotter star but so spotted that it appeared to be dimmer. Since star 1 is, nevertheless, cooler than the more massive component in most Algols, he identified RT Lacertae as one of a small number of 'cool Algols' (Popper 1992). Most recently, Lanza et al. (2002) modeled the variation in system light as found in the Turkish archival data with spot optimization codes derived from an application of maximum entropy and Tikhonov methods, despite the ill-conditioned nature of the problem (illustrated by differences in high latitude spot coverage between the two methods). While not carrying out a full independent analysis of system and stellar parameters, these authors found some consistency with Eaton \& Hall parameters.

We begin with a summary of the properties of this complicated system.

\section{Properties of the System}

\subsection{Basic Properties and Characteristics}

\begin{tabular}{|c|c|c|}
\hline Type & Property & Investigator(s) \\
\hline Period: & 5:073945 d; variable $q$ & Lanza et al. (2002) \\
\hline \multirow{3}{*}{ L.C.: } & Asymmetric maxima & Wachmann (1935) \\
\hline & Variable minima & $\begin{array}{l}\text { Wachmann (1935); } \\
\text { Himpel (1936) }\end{array}$ \\
\hline & Blue primary minimum & Milone (1968) \\
\hline \multirow[t]{2}{*}{ Sp. Types: } & G9 IV, K1: IV & $\begin{array}{l}\text { Joy }(1931) \\
\text { Milone }(1976)\end{array}$ \\
\hline & G9 IV (or G8 III), G5::(III) & Popper (1991) \\
\hline \multirow{2}{*}{ Masses $\left(\mathrm{M}_{\odot}\right)$} & $0.6,1.5$ & Popper (1975) \\
\hline & $.78 \pm .03,1.66 \pm .08$ & Huenemoerder \& Barden (1986) \\
\hline \multirow[t]{2}{*}{ Radii $(a)$ : } & $\begin{array}{l}.31, .27, .26 ; .26, .25, .25 \\
\text { non-luminous occulting area ra }\end{array}$ & $\begin{array}{l}\text { Milone }(1977) \\
\text { adii: }[0.32,0.31]\end{array}$ \\
\hline & $0.295,0.270$ & Eaton \& Hall (1979) \\
\hline \multirow[t]{3}{*}{ Emission: } & $\mathrm{H} \& \mathrm{~K}$ & $\begin{array}{l}\text { Joy (1931); } \\
\text { Droppo \& Milone (1974) }\end{array}$ \\
\hline & $\mathrm{H} \alpha, \mathrm{H} \beta$ & Huenemoerder (1985) \\
\hline & $\begin{array}{l}\text { UV: Mg h \& k, Nv, Si II, } \\
\text { Si IV, O I, C II \& C IV }\end{array}$ & Huenemoerder (1988) \\
\hline X-Ray: & high soft-X-ray luminosity & Walter \& Bowyer (1981) \\
\hline & Excesses (e.g. $0.4 \leq \delta K \leq 0.7$ ) & Milone (1976) \\
\hline Radio: & $L \approx 10 \times$ normal RS CVn & Owen \& Gibson (1978) \\
\hline
\end{tabular}




\subsection{Further Characterization}

Milone (1976) presents evidence of phase-independent (peaking at $\approx 2 \mu \mathrm{m}$, implying $T=1500 \mathrm{~K}$ ) and phase-dependent (peaking at $\phi \approx 0.6) J H K L$ excesses. The phase-dependent excesses can be explained in principle by spot areas of the right temperature but not the constant part, which requires $80 \%$ spot coverage of star 1 and $50 \%$ of star 2, according to Lanza et al. (2002). It is noteworthy that the excesses measured in 1972 were close to maximum at the occultation eclipse of star 1 , when the photosphere of that star was invisible. The analysis assumed $A_{V}=0.21$, and G9 IV and K1 IV stars, and so is conservative, but more complete data and fuller analyses are needed to confirm the magnitudes of the excesses.

The $B-V, U-B$ blue 'PM', and far UV excess imply that star 1 may be intrinsically bluer (\& brighter in the far UV), with a corresponding spectral type as early as G8 (Huenemoerder \& Barden 1986) or even G3-G4 (Ibanoglu et al. 2001), but the latter increases the sizes of the apparent IR excesses; alternatively some of the UV is due to CSM scattered light.

The X-Ray and radio luminosities are higher than for RS CVn-type systems (Walter \& Bowyer 1981; Owen \& Gibson 1978). This suggests there may be transient stream effects, as well as transient active regions.

$\mathrm{H}$ emissions are seen at the systemic velocity with a dispersion of $260 \mathrm{~km} / \mathrm{s}$ (Huenemoerder 1988) and so not associated with either component - indicative of circumstellar/system effects resulting from stream impact splash.

All modeling suggests that RT Lac is close to being semi-detached, with star 2 at $\sim 90 \%$ or more of its Roche lobe. Recent modeling of the 1965 data by the author indicates the semi-detached model to be viable. In any case, work by Tout \& Hall (1991) shows that wind-driven exchange can be important when the mass-losing star merely approaches its lobe. Thus there is every expectation of mass exchange, and it is indeed seen.

Hydrodynamic simulations reveal impacting speeds at $\sim 500 \mathrm{~km} / \mathrm{s}$ on trailing edge of star 1, high excitation UV lines are seen, and $\mathrm{H}$ absorption sometimes are seen to be stronger at Max I (when impact site might be visible). These observations imply evidence of at least transient stream impact effects (Huenemoerder \& Barden 1986). Terrell (private correspondence 2002), with a newer code, confirms significant effects of an impact of stream material on star 1 .

If star 1 is the hotter component, as most studies suggest, the mass ratio ( $\sim 0.4$ ) suggests that RT Lac is a 'cool Algol' system, for which the slope of the $M-T_{\text {eff }}$ relation is opposite to that of classical Algols (Popper 1991, 1992; Torres, Neuhäuser, \& Wichmann 1998). Both components are evolved (Gunn, Mitrou, \& Doyle 1998) and the initial mass ratio is much closer to unity than in classical Algols. Barrado et al. (1994) claim that the components are not on the same isochrone; however, their Table 1 has the properties of the components conflated, so their conclusions about RT Lac may be invalid.

\section{Models}

While there are essentially two variant models of this system (star 1 or star 2 as the hotter component), they differ additionally in having been treated in 
different ways and with different codes. The treatments differ in the use of spots, the use of 'atmospheric eclipses', and in physical geometry. See Kallrath \& Milone (1999) for the methods and the corresponding binary star models which each incorporates. Basic modeling results are summarized in Table 1.

\begin{tabular}{|c|c|c|c|c|c|c|}
\hline Modeler [Model] & Method & $x$ & $T_{M_{1}}$ & $L_{M_{2}}$ & $L_{M_{1}}$ & $\bar{k}$ \\
\hline Fowler (1920) & Russell & $\begin{array}{l}1 \\
0\end{array}$ & $\mathrm{C}$ & .585 & .415 & $\begin{array}{l}1.00 \\
1.00\end{array}$ \\
\hline Krat \& Nekrasova (1936) & Russell & $\begin{array}{c}1 \\
0 \\
0.5\end{array}$ & $\begin{array}{l}\mathrm{C} \\
\mathrm{C} \\
\mathrm{C}\end{array}$ & $\begin{array}{l}.687 \\
.623 \\
.655\end{array}$ & $\begin{array}{l}.313 \\
.377 \\
.345\end{array}$ & $\begin{array}{l}0.70 \\
0.86 \\
\ldots\end{array}$ \\
\hline Milone $(1976,1977)$ & $\begin{array}{l}\text { Russell-Merrill } \\
{\left[T_{M_{1}}=4840 ; T_{M_{2}}\right.} \\
\text { dark envelope arour }\end{array}$ & $\begin{array}{c}0.8 \\
=5155, \text { with } K \\
\text { nd } M_{1} ; \text { const. }\end{array}$ & $\begin{array}{l}\mathrm{C} \\
1 \mathrm{IV}+\mathrm{G} \\
\text { - phase- }\end{array}$ & $\begin{array}{l}.588 \\
\text { var. IR }\end{array}$ & $\begin{array}{c}.412 \\
\text { excess] }\end{array}$ & 0.92 \\
\hline Eaton \& Hall (1979) [s] & $\begin{array}{l}\text { Wilson-Devinney } \\
{\left[T_{M_{1}}=5715 \pm 50\right.} \\
\text { ellipticity, radius ch }\end{array}$ & $\begin{array}{c}0.7,0.82 \\
T_{M_{2}}=5300 ; T \\
\text { langes of star } 1\end{array}$ & $\stackrel{\mathrm{H}}{p o t s}=0$ & .531 & 469 & 0.92 \\
\hline Lanza et al. (2002) [s] & $\begin{array}{l}\text { WD/new? } \\
{\left[T_{M_{2}}=5100 ; T_{M_{2}}\right.} \\
\text { no ellipticity or rad } \\
\text { spot area: star } 1:\end{array}$ & $\begin{array}{r}\text { u: } 0.76,0.79 \\
\text { s: } 0.82,0.83 \\
=4700 ; T_{\text {spots }} \\
\text { ius changes we } \\
40 \% \& \text { var; s }\end{array}$ & $\begin{array}{l}\mathrm{H} \\
\text { 1000; } \\
\text { found; } \\
\text { ar 2: }\end{array}$ & .606 & .394 & 0.98 \\
\hline
\end{tabular}

The quantity $x$ refers to the linear limb-darkening coefficient; $T_{M}$ refers to the temperature of the more massive star (star 1 ); $L$ refers to relative luminosity; $k$ is the ratio of radii (always taken as less than one); $C$ indicates that $M_{1}$ (star 1$)$ is assumed to be the cooler star. The symbols $[\mathrm{s}]$ and [u] indicate spot and non-spot models or regions.

Useful discriminators for physical models for RT Lac are:

1. a quantitative explanation for the late spectral classification of the more massive star. This is a test also of system models, especially the radii (see below);

2. evidence of molecular absorption on either component, and useful to provide spot temperatures;

3. direct evidence of magnetic fields, both globally, and in active regions. These can test the mechanism of the relationship of the stellar dynamos to period changes and light curve variations (Lanza, Rodono, \& Rosner 1998; Lanza et al. 2002);

4. the spectral behaviour of the 'migrating wave', which is problematic if not due to activity cycles;

5. the varying signs of determined period changes (Ibanoglu et al. 2001; Lanza et al. 2002), which are somewhat problematic if not caused by activity cycles; a coupling mechanism has been proposed involving stellar magnetic dynamos and quadrupole moments of the components (Applegate 1992; Lanza et al. 1998).

\section{Discussion and Future Work}

All authors prior to Popper (1991) referred to star 1, which is in front at the the (usually) deeper minimum (therefore assumed to be primary minimum), as having spectral type K1IV, based on direct examination of stellar spectra at this phase. Popper believed that Joy's K1IV classification was incorrect since 
the long exposure plates of that era could not have avoided having the spectra of the two stars blended to some extent. There is no disagreement that the eclipsing star is the more massive, however, and on this basis, it is designated 'star 1' here. For star 1, Popper suggested G5, the spectral class listed in the Henry Draper Catalogue. The less massive component, 'star 2', that Joy and Milone both classified as G9IV, in fact dominates the spectrum at all phases save primary minimum. Popper (1991) preferred G8 for star 2 and its photometrically derived dimensions made it a giant. Milone (1977) indicated that the spectrum of star 1 , seen at phase zero, the deeper $V$ band minimum in the light curves of that epoch, could not be earlier than K0IV nor later than K1IV (in a DAO spectrogram of 21-min exposure). A reexamination of all RT Lac spectra in the author's possession confirms that there is no evidence of a much earlier spectrum at any phase. Spectra at the (usually) shallower minimum do not show a significantly later spectral type either, so one cannot attribute the late PM spectrum to gravity darkening effects in photospheres, alone. The spectrum of star 2, the larger and more luminous star in UBV passbands, is seen to be G8-G9 on the basis of the relative strengths of the Cr 4254 and 4274 lines relative to nearby iron lines. Popper did not have spectra at either minimum, and found that he could not discern the spectral type of star 1 outside eclipse, so he must have adopted G5 on the basis of light curve and colour index data obtained at primary minimum, as he seems to indicate (Popper 1991). Thus his assessment is compromised to some degree by circumstellar and/or interstellar reddening. Nevertheless, Popper's intuition was correct: contamination with a G8 or G9 star can produce a later spectral type given the right eclipse circumstances. Model runs by the author indicate that even at central transit in the 1965 light curve, for instance, star 1 contributed only $\sim 40 \%$ of the system light near $\lambda 4400$ and at $\Delta \phi=0.03$ away from this minimum contributed less than a third. The brightness of the system, and presumably of star 1 , varies with time, so the model cannot be applied rigidly to other epochs, but qualitatively this explains why it has been so difficult to observe an earlier spectral type at deep minimum. The K0 or K1 classification may therefore arise from the transit-selected limbdarkened regions of star 2 (normally outshown by the central portions of its disk). This means that higher resolution spectra at quadratures are required to separate the components' lines unless the photospheric surface is relatively unobscured. In one spectrogram at $\sim 30 \AA / \mathrm{mm}$, DAO plate 76913 taken at HJDN 2442286.8040, when the phase (reckoned from the transit minimum) was $\sim 0.21$, the ratio of $\mathrm{Cr} 4254$ ( 4274 is blended) to nearby Fe lines is nearly equal in strength in the two stars; for stars around $\mathrm{K} 0$ the ratio is different from earlier types. Additional spectra with higher $\mathrm{S} / \mathrm{N}$ and resolution are needed to confirm this indication.

If the UV excesses of star 1 are explicable by scattered UV emission off an envelope, no one has demonstrated in detail that such a model works quantitatively and most have preferred to consider that its surface is masked by either a circumstellar envelope or by a massive band of dark spots. The Turkish archival photoelectric light curves, averaged over seasons, do show that the minima reverse, and that the overall brightness of the system does change, over intervals of years. The author's own data show that light curve changes are seen over even shorter intervals. While spot cycle mechanisms are known, there has been no consensus as to what could cause an envelope's opacity to vary cyclically 
on times scales of years. One possibility, however, is that star 2's mass loss is modulated by active cycle winds on that star.

The bulk of the evidence suggests spots to be the main cause of optical light curve anomalies, and that these spots are mainly located on star 1 . In the RT Lac system, star 1, the smaller and assumedly hotter star, is assumed to be spotted to a greater degree than its cooler and larger companion, unlike RS CVn-like systems, but like other 'cool Algols' (e.g. Torres et al. 1998). Moreover, velocities of some spectral lines, large radio flux, and apparent IR excesses all suggest the presence of material not confined to either component. Light curve modelers to the present have not analyzed non-optical observations along with the optical and so have not taken account of the effects of material splashed from the stream impact. Recent preliminary modeling by the author suggests that a semi-detached model may be viable and must be explored along with detached models in any future analyses. Analyses of pre-photoelectric data convinced Hall \& Haslag (1976) long ago that one of the radii changed with time. The determination of the radii are therefore critical to understand this evolving system.

Sorting out the mechanisms requires more than fiat assumptions: it requires detailed model comparisons. These require more data, simultaneous and synoptic, to assess the relative effects of active regions, streams, and gas: coordinated, multi- $\lambda$ photometry, polarimetry, and high-dispersion spectroscopy. The most critical parts are achievable with new instrumentation, e.g., ESPaDOnS (Echelle Spectropolarimetric Device for the Observation of Stars), which is scheduled for first light on the CFHT in 2003. This versatile, broad-band $(0.37-1.00 \mu \mathrm{m})$, high resolution $(\lambda / \Delta \lambda=50,000)$, polarimetry instrument may provide evidence of molecular features in heavily spotted regions, the orientations and strengths of magnetic field lines, and detailed radial velocity data. So that structures can be associated with specific light curve effects, multi- $\lambda$ photometry must be carried out simultaneously.

Finally, to make best use of new as well as archival data, we must use modeling codes that permit treatment of polarization data, colour indices, atmospheric 'clouds', disks, and photospheric spots. These are now features of Wilson-Devinney programs, the engines of which have damped least squares, with at least one version (WD2002) having auto-iteration. Spot optimization is improving, and various advanced treatments are in use in other codes.

Collaborators in all aspects of this work, are, of course, welcome. With this attack, a fully satisfactory solution for RT Lacertae should be be within reach.

Acknowledgments. Some of the work in this paper was supported by NSERC of Canada grants; the aid of the SOC and LOC in securing partial travel funding from the IAU is acknowledged with gratitude.

\section{References}

Applegate, J. H. 1992, ApJ, 385, 621

Barrado D., Fernandez-Figueroa M. J., Montesinos B., \& De Castro E. 1994, A\&A, 290, 137

Eaton, J. A., \& Hall, D. S. 1979, ApJ, 227, 907 
Gunn, A. G., Mitrou, C. K., \& Doyle, J. G. 1998, MNRAS, 296, 150

Hall, D. S. 1968, IBVS, 259

Hall, D. S. 1976, in IAU Coll. 29, Multiple Periodic Variable Stars, Part I, ed. W. S. Fitch (Dordrecht: Reidel), 287

Hall, D. S. \& Haslag, K. P. 1976, in IAU Coll. 29, Multiple Periodic Variable Stars, Part II, ed. W. S. Fitch (Budapest: Academic Press), 331

Himpel, K. 1936, AN, 261, 254

Huenemoerder, D. P. 1985, AJ, 90, 499

Huenemoerder, D. P. 1988, PASP, 100, 600

Huenemoerder, D. P., \& Barden, AJ, 91, 583

Ibanoglu, S., Evren, S., Tas, G., Devlen, A., \& Cakirh, O. 2001, A\&A, 371, 626 Johnson, H. L. 1966, ARA\&A, 4, 193

Joy, A. H. 1931, ApJ, 74, 101

Kallrath, J., \& Milone, E. F. 1999, Eclipsing Binary Stars: Modeling and Analysis (New York: Springer-Verlag)

Krat, W., \& Nekrasova, S. 1936, Acta Astron. Ser. C, 2, 129

Lanza, A. F., Catalano, S., Rodonò, M., Ibanoglu, C., Evren, S., Tas, G., Çakirli, Ö., \& Devlen, A. 2002, A\&A, 386, 583

Lanza, A. F., Rodono, M., \& Rosner, R. 1998, MNRAS, 296, 893

Luizet, M. 1910, Bull. Astronomique, 27, 302

Luizet, M. 1915, Bull. Astronomique, 32, 68

Milone, E. F. 1968, AJ, 73, 708

Milone, E. F. 1976, ApJS, 31, 93

Milone, E. F. 1977, AJ, 82, 998

Nijland, A. A. 1917, AN, 204, 65

Nijland, A. A. 1920, AN, 211, 361

Owen, F. N., \& Gibson, D. M. 1978, AJ, 83, 1488

Popper, D. M. 1991, AJ, 101, 220

Popper, D. M. 1992, in IAU Symp. 151, Evolutionary Processes in Interacting Binary Stars, ed. Y. Kondo, R. F. Sistero, \& R. S. Polidan (Dordrecht: Kluwer), 395

Popper, D. M. 1996, ApJS, 106, 133

Torres, G., Neuhäuser, R., \& Wichmann, R. 1998, AJ, 115, 2028

Tout, C. A., \& Hall, D. S. 1991, MNRAS, 253, 9

Wachmann, A. A. 1935, AN, 255, 341

Walter, F. M., \& Bowyer, S. 1981, ApJS, 53, 815 